\title{
EXTREMITY EXPOSURE IN NUCLEAR MEDICINE: PRELIMINARY RESULTS OF A EUROPEAN STUDY
}

\author{
M. Sans Merce ${ }^{1, *}$, N. Ruiz ${ }^{1}$, I. Barth ${ }^{2}$, A. Carnicer ${ }^{3}$, L. Donadille ${ }^{4}$, P. Ferrari ${ }^{5}$, M. Fulop ${ }^{6}$, M. Ginjaume ${ }^{3}$, \\ G. Gualdrini ${ }^{5}$, S. Krim ${ }^{7}$, F. Mariotti ${ }^{5}$, X. Ortega ${ }^{3}$, A. Rimpler ${ }^{2}$, F. Vanhavere ${ }^{7}$ and S. Baechler ${ }^{1}$ \\ ${ }^{1}$ Institute of Radiation Physics, University Hospital Center of Lausanne, Grand-pré 1, 1007 Lausanne, \\ Switzerland \\ ${ }^{2}$ Bundesamt für Strahlenschutz, Köpenicker Allee 120-130, 10318 Berlin, Germany \\ ${ }^{3}$ Universitat Politecnica de Catalunya (UPC), Diagonal 647, 08028 Barcelona, Spain \\ ${ }^{4}$ Institut de Radioprotection et de Sûreté Nucléaire (IRSN), BP17, 92262 Fontenay-aux-Roses, France \\ ${ }^{5}$ Ente per le Nuove Tecnologie, l'Energia e l'Ambiente (ENEA), Via dei Colli 16, 40136 Bologna, Italy \\ ${ }^{6}$ Slovak Medical University, Limbova 12, 83303 Bratislava, Slovakia \\ ${ }^{7}$ Belgian Nuclear Research Centre (SCK•CEN), Boeretang 200, 2400 Mol, Belgium \\ *Corresponding author: marta.sans-merce@chuv.ch
}

\begin{abstract}
The Work Package 4 of the ORAMED project, a collaborative project (2008-11) supported by the European Commission within its seventh Framework Programme, is concerned with the optimisation of the extremity dosimetry of medical staff in nuclear medicine. To evaluate the extremity doses and dose distributions across the hands of medical staff working in nuclear medicine departments, an extensive measurement programme has been started in 32 nuclear medicine departments in Europe. This was done using a standard protocol recording all relevant information for radiation exposure, i.e. radiation protection devices and tools. This study shows the preliminary results obtained for this measurement campaign. For diagnostic purposes, the two most-used radionuclides were considered: ${ }^{99 \mathrm{~m}} \mathrm{Tc}$ and ${ }^{18} \mathrm{~F}$. For therapeutic treatments, Zevalin ${ }^{\circledR}$ and DOTATOC (both labelled with ${ }^{90} \mathrm{Y}$ ) were chosen. Large variations of doses were observed across the hands depending on different parameters. Furthermore, this study highlights the importance of the positioning of the extremity dosemeter for a correct estimate of the maximum skin doses.
\end{abstract}

\section{INTRODUCTION}

As a consequence of the definition that the dose limit for the skin has to be applied to 'the dose averaged over any area of $1 \mathrm{~cm}^{2}$ regardless of the area exposed', it is advisable to measure the local skin dose at the location with presumably the highest exposure. This requirement is the central dilemma of extremity dosimetry and causes severe practical difficulties. In daily practice, in nuclear medicine, it is often not known which part of the hand will receive the highest dose. Moreover, the dose distribution over the hand may vary during a single process as well as when several people perform the same procedure ${ }^{(1)}$. To tackle, amongst other things, these problems, the ORAMED project (a collaborative project supported by the European Commission within its seventh Framework Programme) was started at the beginning of 2008. The Work Package 4 of this project aims at optimising radiation protection of medical staff in nuclear medicine. The goal of the present work was to measure skin doses $H_{\mathrm{p}}(0.07)$ at different positions on the hands for several nuclear medicine procedures using the appropriate measuring tools. The procedures were selected according to their frequency of use, the delivered doses and the three types of radionuclides commonly used in nuclear medicine, i.e. positron, beta and gamma emitters.. For diagnostic purposes, two radionuclides are considered: ${ }^{18} \mathrm{~F}$ is used for positron emission tomography (PET) examinations and ${ }^{99 \mathrm{~m}} \mathrm{Tc}$ is used for planar and single photon emission computed tomography (SPECT) examinations. The two most frequently used therapeutic agents are ${ }^{90} \mathrm{Y}$ labelled Zevalin ${ }^{\circledR}$ and DOTATOC. Other treatments based on drugs labelled with other radionuclides ${ }^{32} \mathrm{P}$, ${ }^{177} \mathrm{Lu}$, etc.) were also registered, but the results will not be reported in this paper.

\section{MATERIALS AND METHODS}

An extensive measurement programme is being performed in 32 nuclear medicine departments distributed among hospitals in seven different countries across Europe with a unified protocol, where all relevant information for radiation exposure (i.e. radiation protection devices and tools, dominant hand, staff experience) were recorded. Using the same protocol, the measurements are harmonised and all data can be compared and evaluated. The protocol includes preparation and administration, independently, both for diagnostic and therapeutic applications. The skin dose on the hands was measured with high-sensitivity thermoluminescent dosemeters (TLDs) specific for beta- and gamma-radiation. Figure 1 shows their placement on gloves at 11 different positions for each hand.. Moreover, TLDs 
were welded in thin plastic bags to reduce the thickness on top of the TLD and adequately measure the quantity $H_{\mathrm{p}}(0.07)$.

An intercomparison between the participating laboratories was performed at IRSN to assess and compare the methods used for the calibration of the dosemeters. The main goal of this intercomparison was to establish a common basis for the measurement campaign among all participants. Two different reference irradiation fields were used: ${ }^{137} \mathrm{Cs}$ and ${ }^{85} \mathrm{Kr}$, defined in compliance with the ISO 4037-1 ${ }^{(2)}$ and $6980-1^{(3)}$ standard. Standard TLDs (LiF:Mg:Cu:P and LiF:Mg:Ti, up to $240 \mathrm{mg} \mathrm{cm}^{-2}$ ), used for gamma-ray measurements, were irradiated with ${ }^{137} \mathrm{Cs}$ and thin TLDs $\left(8-10 \mathrm{mg} \mathrm{cm}^{-2}\right)$, used for positron and beta pharmaceuticals ${ }^{(4)}$, were irradiated with ${ }^{85} \mathrm{Kr}$. All participants reported results within $10 \%$ of the reference value $(2 \mathrm{mSv})$.

\section{RESULTS AND DISCUSSION}

Preliminary results from the measurement campaign are shown in Tables 1 and 2. At the moment, 115 workers from 32 nuclear medicine departments have been monitored. The aim is that every worker is monitored at least five times for the same diagnostic procedure. For this study, all measurements have been considered even when the number is smaller than five (for $50 \%$ of the workers). To compare measurements from different departments, for each procedure, the dose equivalent to the hands $H_{\mathrm{p}}(0.07)$ was measured and normalised to the respective manipulated activity. In general, there is a large spread of results, due to the influence of many factors, such as the type of protections used and the experience of the workers. The complete statistical analysis of the results is under progress. The uncertainties associated to the different values are not given for simplification of reading the tables and figures.

The maximum normalised doses in Tables 1 and 2 correspond to the maximum values among all workers and all measuring positions in the hands. Mean and median dose values correspond to the mean and median value at the position where the maximum dose is most frequently found, usually the index tip/nail and the thumb.

Normalised doses are higher for ${ }^{18} \mathrm{~F}$ than for ${ }^{99 \mathrm{~m}} \mathrm{Tc}$, as shown in Table 1 . However, diagnostic procedures using ${ }^{99 \mathrm{~m}} \mathrm{Tc}$ are more frequent than those using ${ }^{18} \mathrm{~F}$. Thus, the activities manipulated are usually higher for ${ }^{99 \mathrm{~m}} \mathrm{Tc}$ than for ${ }^{18} \mathrm{~F}$. Comparing Table 1 with Table 2, the doses obtained for diagnostics procedures were found to remain much lower than the values measured for therapeutic procedures with ${ }^{90} \mathrm{Y}$. Usually preparation delivers higher doses than the administration of the radiopharmaceutical. Three main reasons contribute to this effect. First, the activities manipulated for preparation are higher
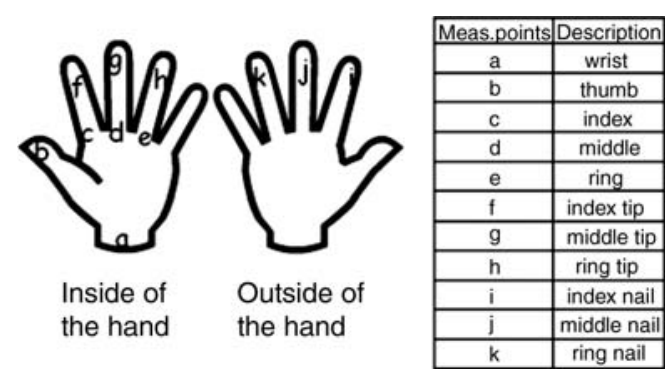

Figure 1. TLDs positioning in the hands.

Table 1. Maximum, median and mean normalised doses for the two radionuclides most commonly used for diagnostics.

\begin{tabular}{lcccc}
\hline & \multicolumn{5}{c}{ Diagnostic radionuclides } \\
\cline { 2 - 6 } & $\mathrm{A}-{ }^{99 \mathrm{~m}} \mathrm{Tc}$ & $\mathrm{P}-{ }^{99 \mathrm{~m}} \mathrm{Tc}$ & $\mathrm{A}-{ }^{18} \mathrm{~F}$ & $\mathrm{P}-{ }^{18} \mathrm{~F}$ \\
\hline Maximum $\left(\mathrm{mSv} \mathrm{GBq}^{-1}\right)$ & 1.50 & 2.06 & 3.67 & 4.43 \\
Median $\left(\mathrm{mSv} \mathrm{GBq}^{-1}\right)$ & 0.07 & 0.17 & 0.33 & 0.64 \\
Mean $\left(\mathrm{mSv} \mathrm{GBq}^{-1}\right)$ & 0.07 & 0.32 & 0.71 & 0.77 \\
\hline
\end{tabular}

'A' stands for administration and 'P' for preparation.

than those manipulated for the administration of the radiopharmaceutical. Second, some of the preparation steps are performed with an unshielded source, while the administration of the radiopharmaceutical to the patient is usually performed with a shielded syringe. Finally, the time needed to prepare a radiopharmaceutical is longer than the time needed for administering it.

\section{Mean normalised doses per position}

The mean normalised doses found for each monitored hand position are shown in Figures 2 and 3 for diagnostic and therapeutic procedures, respectively.

From Figures 2 and 3, it is clearly seen that the tips and nails of the fingers are the most exposed areas in the hands. The two most exposed fingers are the index and the thumb usually of the non-dominant hand, followed by the middle and the ring. The wrist position, even if used in some countries as routine monitoring position ${ }^{(5)}$, is the least exposed position in the hand.

\section{Position of the dosemeter for extremity monitoring}

To comply with the definition of dose limit for the skin, the recommended position for extremity monitoring is the position where the maximum dose can be found. 
EXTREMITY EXPOSURE IN NUCLEAR MEDICINE

Table 2. Maximum, median and mean normalised doses for ${ }^{90} \mathrm{Y}$ Zevalin ${ }^{\circledR}$ and DOTATOC therapies.

\begin{tabular}{|c|c|c|c|c|}
\hline & \multicolumn{4}{|c|}{ Therapeutic radionuclides } \\
\hline & A- ${ }^{90} \mathrm{Y} \mathrm{Zevalin}^{\circledR}$ & P- $-{ }^{90}$ Y Zevalin ${ }^{\circledR}$ & A- ${ }^{90} Y_{\text {Dotatoc }}{ }^{\circledR}$ & P- ${ }^{90} \mathrm{Y}$ Dotatoc ${ }^{\circledR}$ \\
\hline $\operatorname{Maximum}\left(\mathrm{mSv} \mathrm{GBq}^{-1}\right)$ & 11.17 & 32.05 & 6.27 & 5.84 \\
\hline Median (mSv $\left.\mathrm{GBq}^{-1}\right)$ & 2.16 & 2.07 & 0.56 & 0.34 \\
\hline Mean $\left(\mathrm{mSv} \mathrm{GBq}^{-1}\right)$ & 2.46 & 3.52 & 0.65 & 1.65 \\
\hline
\end{tabular}

'A' stands for administration and 'P' for preparation.

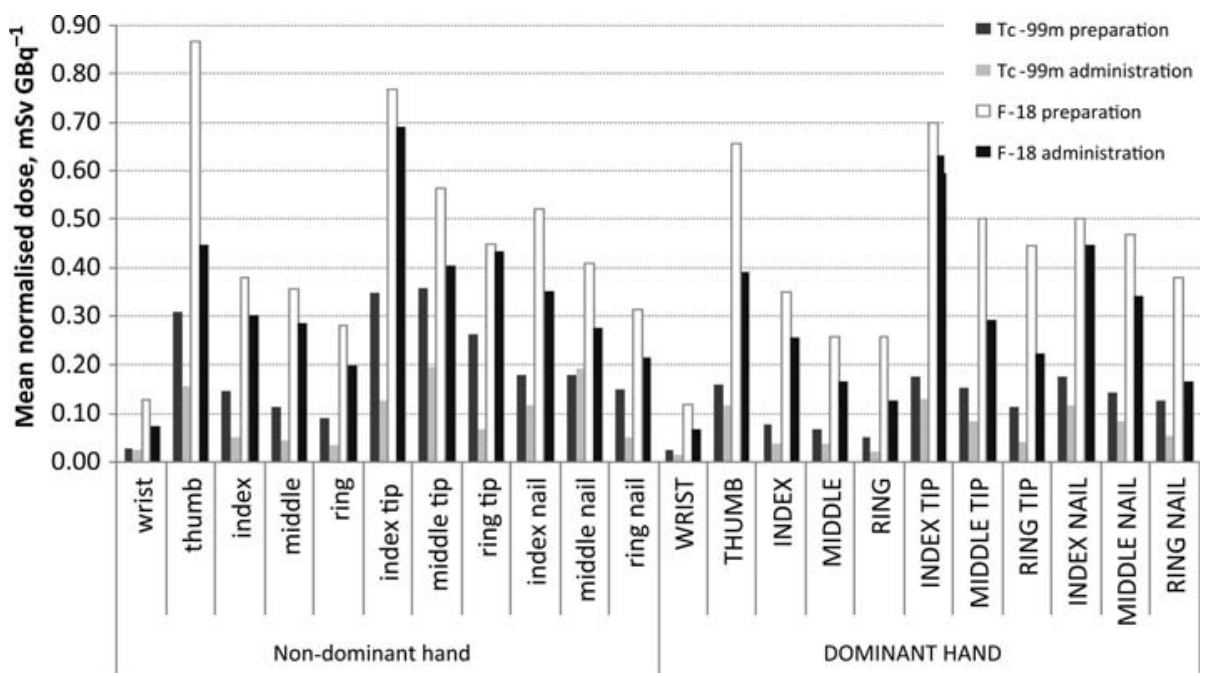

Figure 2. Mean normalised doses in each position of the hands for the radionuclides used in diagnostics and for both procedures - preparation and administration.

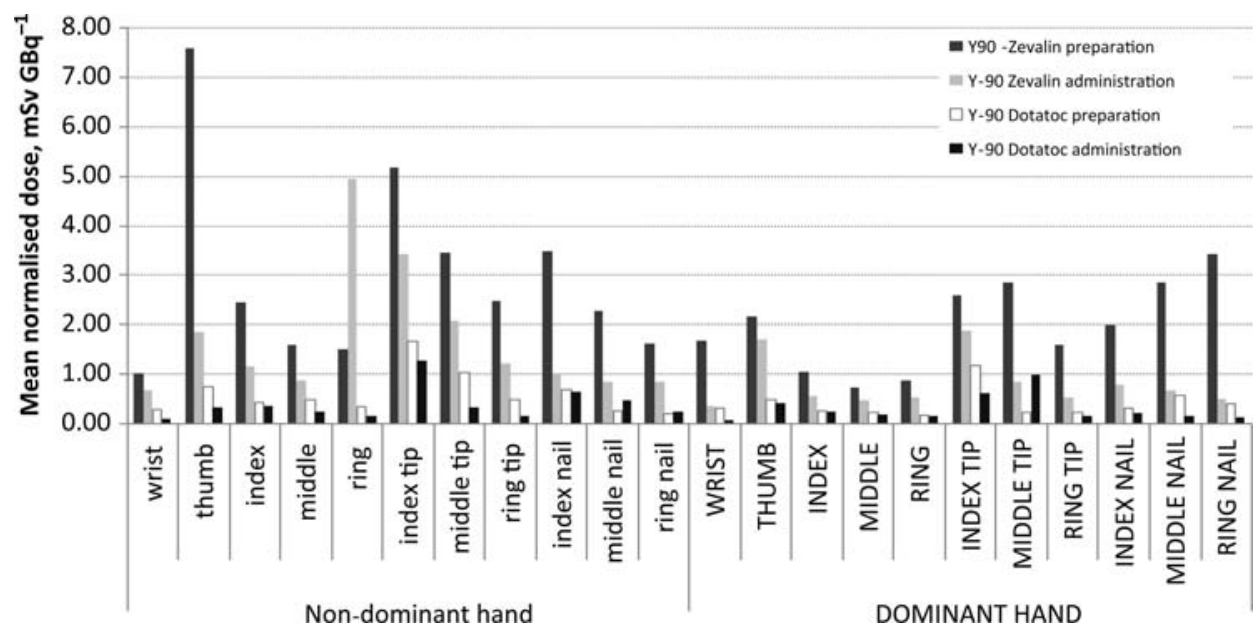

Figure 3. Mean normalised doses in each position of the hands for both preparation and administration for the two therapies (Zevalin ${ }^{\circledR}$ and DOTATOC) considered. 


\section{SANS MERCE ET AL.}
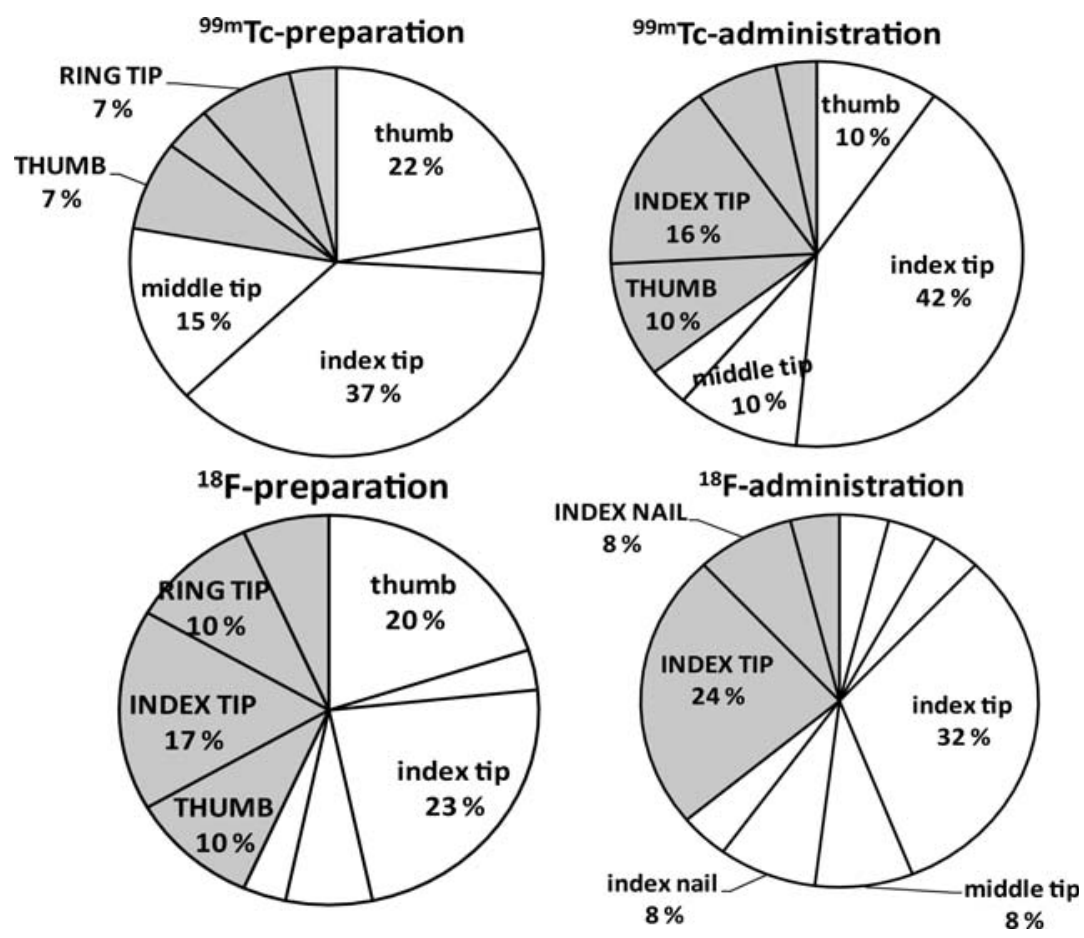

Figure 4. Frequencies of the position where the maximum dose was obtained for preparation and administration of ${ }^{99 \mathrm{~m}} \mathrm{Tc}$ and ${ }^{18} \mathrm{~F}$. (The regions highlighted in grey and with capital letters correspond to the dominant hand, while that in white correspond to the non-dominant hand. Positions where values are under $7 \%$ are not labelled.)

Figures 4 and 5 show for each radionuclide and for each procedure, the frequencies of the position where the maximal dose was measured. The most frequent positions correspond to the index tip/nail and thumb of the non-dominant hand.

The impact of placing the routine monitoring dosemeter at a different position than the one corresponding to the maximal hand dose has been estimated by calculating correction factors as ratios between the index tip or maximum in the hand and the position usually used for routine monitoring, i.e. the base of the index or the ring finger, as shown in Figure 6.

The values used to compute the correction factors are the mean dose values obtained by averaging among all workers; so individual values can be even much higher. Those factors vary from 1 to 7 depending on the radionuclide and the procedure. The correction factors with respect to the ring finger are usually higher than those with respect to the base of the index finger. Indeed, the index finger, specially the tip, is usually one of the most-exposed fingers, in accordance to what has already been observed in the literature ${ }^{(6)}$.

\section{CONCLUSIONS}

Preliminary results show that large variations of skin doses were observed across the hands, from 0.07 to
$32.05 \mathrm{mSv} \mathrm{GBq}^{-1}$, depending on the radionuclide and procedure, but also on the worker and the radiation-protection measures. Further analyses will be done on these influences in the future.

The positioning of the dosemeter used in routine monitoring strongly affects the estimates of the extremity doses meaning that in some cases, the limit on equivalent dose for the skin $\left(0.5 \mathrm{~Sv} \mathrm{y}^{-1}\right)$ could be surpassed. For example, a worker manipulating an annual realistic activity of $1500 \mathrm{GBq}$ of ${ }^{99 \mathrm{~m}} \mathrm{Tc}$ could be receiving an annual dose of $150 \mathrm{mSv}$ at the base of the ring finger; in this case, the dose at the maximum (factor 4 higher) will exceed the annual limit of $500 \mathrm{mSv}$. From the available results, it can be concluded that the position of the dosemeter on the hand is important to obtain a correct estimate of the extremity dose. Neither the wrist nor the ring positions, ordinarily used as routine monitoring positions, are adequate for this purpose. A more correct position will be the tip of the index finger on the non-dominant hand. However, since this is difficult to accomplish in daily practice, a ring dosemeter placed on the base of the index finger of the nondominant hand might be a better alternative. In this case, radiation protection officers must be aware that the measured dose in such case will be around a factor of 2.5 smaller than the maximum skin dose. 


\section{${ }^{90} Y_{-Z e v a l i n}{ }^{\circledR}$-preparation}
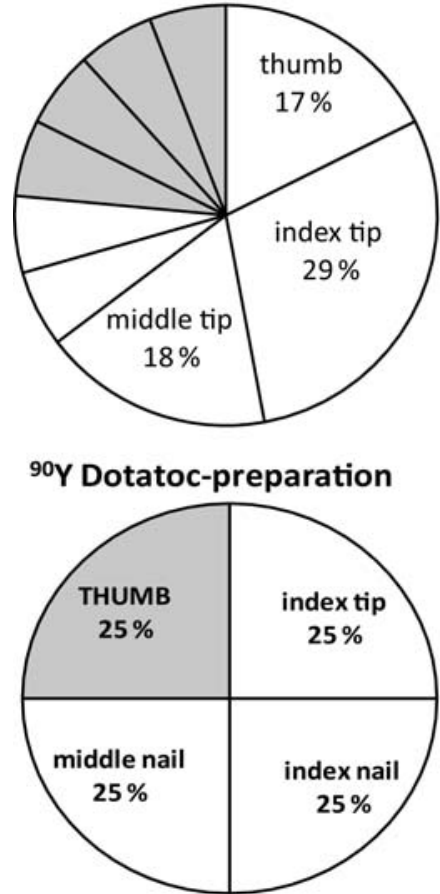

${ }^{90} \mathrm{Y}$ - Zevalin-administration

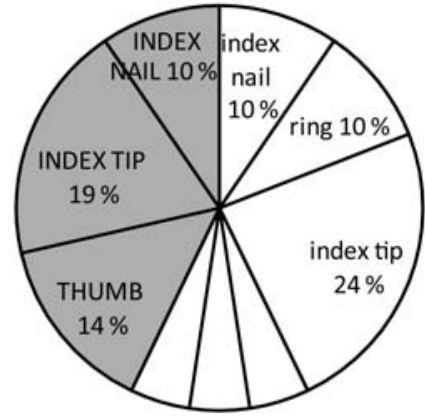

${ }^{90} \mathrm{Y}$ Dotatoc-administration

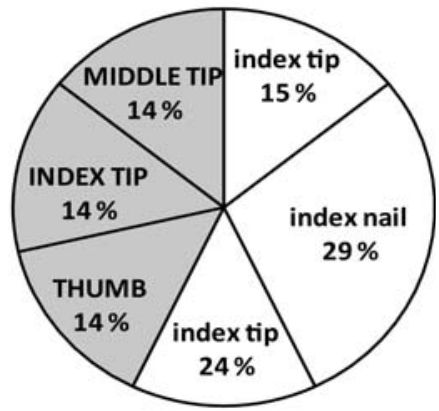

Figure 5. Frequencies of the position where the maximum dose was obtained, for ${ }^{90} \mathrm{Y}$ Zevalin ${ }^{\circledR}$ and ${ }^{90} \mathrm{Y}$ DOTATOC, for preparation and administration. (The regions highlighted in grey and with capital letters correspond to the dominant hand, and white to the non-dominant hand. Positions where values are under $7 \%$ are not labelled.)

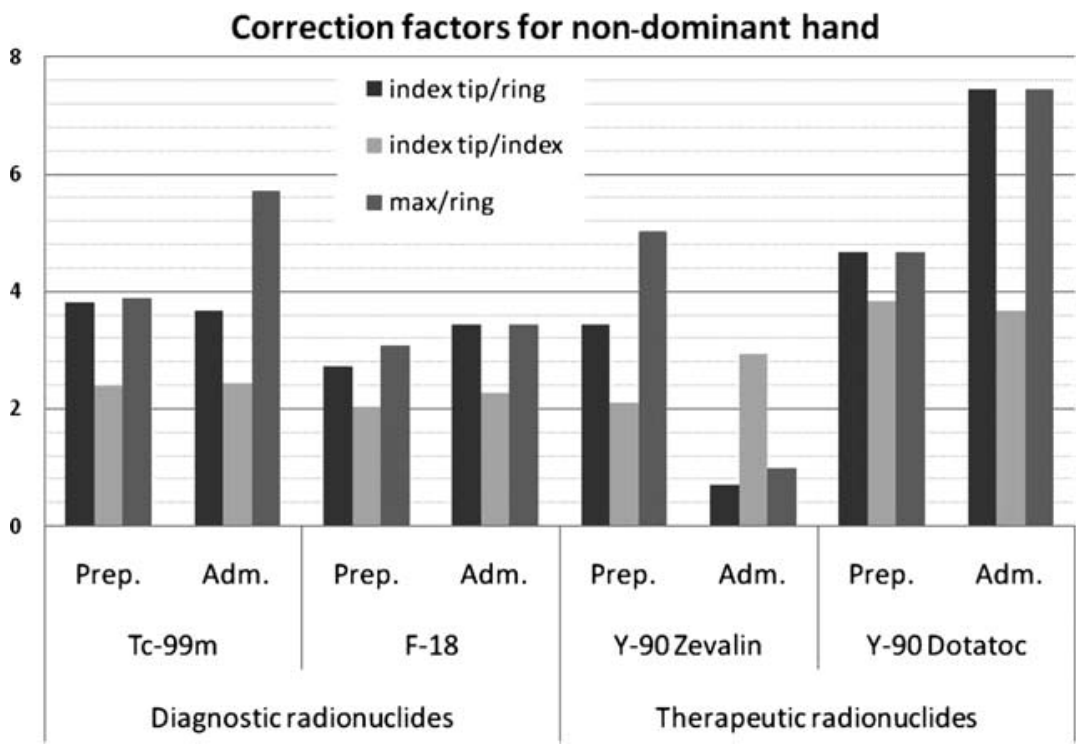

Figure 6. Correction factors computed for the non-dominant hand, i.e. usually the most-exposed hand. 'Prep.' stands for preparation and 'Adm.' for administration. 


\section{SANS MERCE ET AL}

\section{FUNDING}

This work was supported by the European Atomic Energy Community's Seventh Framework Programme (FP7/2007-2011) under grant agreement no. 211361.

\section{REFERENCES}

1. Vanhavere, F., Carinou, E., Donadille, L., Ginjaume, M., Jankowski, J., Rimpler, A. and Sans Merce, M. An overview of extremity dosimetry in medical applications. Radiat. Prot. Dosim. 129, 350-355 (2008).

2. International Organization for Standardization. $X$ and gamma reference radiation for calibrating dosemeters and dose rate meters and for determining their response as a function of photon energy-part I: characteristics of the radiations and their methods of production. ISO 4037-1 (ISO) (1996).

3. International Organization for Standardization. Nuclear energy - reference beta-particle radiation - part 1: method of production. ISO 6980-1 (ISO) (2006).

4. Ginjaume, M. et al. Extremity ring dosimetry intercomparison in reference and workplace fields. Radiat. Prot. Dosim. 131, 67-72 (2008).

5. Donadille, L., Carinou, E., Ginjaume, M., Jankowski, J., Rimpler, A., Sans Merce, M. and Vanhavere, F. An overview of the use of extremity dosemeters in some European countries. Radiat. Prot. Dosim. 131, 62-66 (2008).

6. Wrzesien, M., Olszewski, J. and Jankowski, J. Hand exposure to ionising radiation of nuclear medicine workers. Radiat. Prot. Dosim. 130, 325-330 (2008). 